\title{
Utilidad de la colangiopancreatoscopia directa en el manejo del cálculo pancreático, a propósito de un caso (con video)
}

\section{Usefulness of Direct Cholangiopancreatoscopy for Management of Pancreatic Calculi: Case Study (with video)}

\author{
Martín Alonso Gómez, ${ }^{\text {* }}$ Óscar Fernando Ruiz, ${ }^{2}$ Hernando Marulanda Fernández. ${ }^{3}$
}

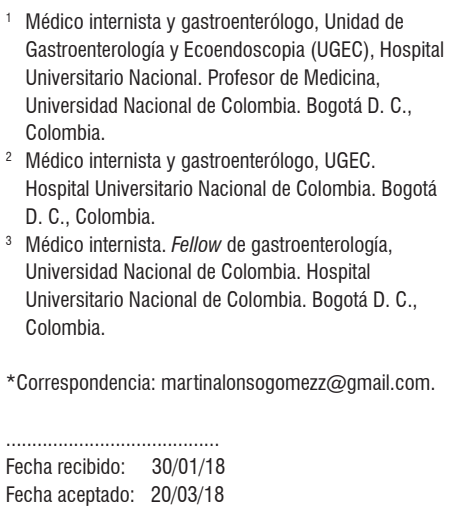

\begin{abstract}
Resumen
Se presenta el caso de un paciente con pancreatitis crónica y hallazgo de lesión quística en el ducto pancreático principal, a quien se le realizó colangioscopia directa tipo Spyglass y se encontró un gran cálculo impactado a nivel de la cabeza del páncreas, condicionando una formación pseudoquística. Se realizó litotricia láser y se logró la extracción en su totalidad, con lo cual se logró la remisión sintomática.
\end{abstract}

\author{
Palabras clave \\ Colangioscopia, Spyglass, pseudoquiste, litotricia.
}

\section{Abstract}

We present the case of a patient with chronic pancreatitis. After finding a cystic lesion in the main pancreatic duct, the patient underwent direct cholangioscopy using the SpyGlass DS System. A large impacted calculus and pseudocyst formation was found in the head of the pancreas. Laser lithotripsy was used to extract the entire calculus and symptomatic remission was achieved.

\section{Keywords}

Cholangioscopy, Spyglass, pseudocyst, lithotripsy.

\section{INTRODUCCIÓN}

La colangioscopia directa tipo Spyglass ha revolucionado el tratamiento de las enfermedades biliopancreáticas (1), facilitando el abordaje de múltiples patologías previamente consideradas eminentemente quirúrgicas, tal y como lo son el cálculo difícil, las estenosis biliares y pancreáticas, y valoración y drenaje de lesiones quísticas pancreáticas (2). No se trata solo de la posibilidad de canular de forma selectiva y realizar una visualización directa de la vía biliar o el ducto pancreático, sino también la opción de pasar una sonda láser con el objetivo de fracturar los cálculos o incluso una pinza de biopsia y tomar las muestras necesarias (3).

El tratamiento de la pancreatitis crónica es muy complejo y más aún cuando el paciente tiene cálculos pancreáticos, ya que estos son muy difíciles de tratar (4). Aunque el manejo más recomendado es la litotricia extracorpórea, esta se usa muy poco en nuestro medio (5), debido a que solo está disponible para las patologías del tracto urológico (6), por lo que el método más usado es la colangiopancreatografía retrógrada endoscópica (CPRE). Este procedimiento tiene unas tasas de éxito cercanas al $60 \%$ en la extracción de cálculos pancreáticos (7), las características propias del lito como su forma y ubicación van de la mano de la posibilidad de éxito terapéutico y de sus complicaciones (8).

\section{CASO CLíNICO}

Se trata de un hombre de 38 años con historia de pancreatitis crónica, cuyos principales síntomas descritos eran dolor 
abdominal y deposiciones diarreicas, con fracaso en múltiples intervenciones farmacológicas, entre ellas enzimas pancreáticas, neuromoduladores y opioides. Durante el seguimiento se documenta en una colangiorresonancia la presencia de un cálculo de $15 \mathrm{~mm}$ asociado con una dilatación sacular del Wirsung en su porción proximal (Figura 1 y Video 1). Se planteó inicialmente manejo quirúrgico, el cual fue rechazado por el paciente, motivo por el cual es remitido. Por ende, se decidió llevar a pancreatoscopia directa tipo Spyglass y litotricia láser.

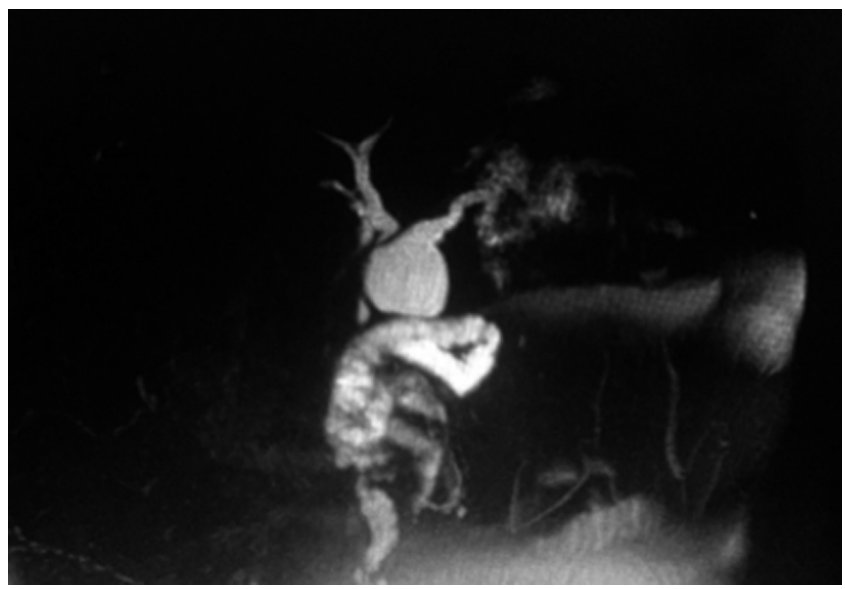

Figura 1. Formación pseudoquística en ducto pancreático principal, secundario a cálculo impactado.

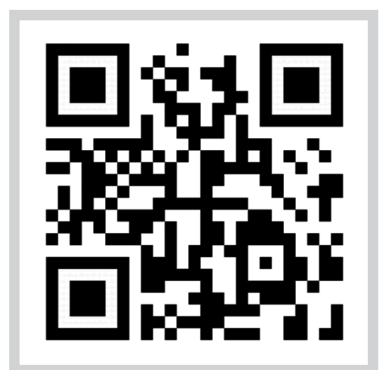

\section{Escanee el código con su smartphone}

Video 1. En el video se observa la colangiorresonancia de un paciente con dilatación severa del Wirsung y un cálculo en su interior. https:// youtu.be/u7rG7WG50To

Para esto se realizó una CPRE con canulación selectiva de la vía pancreática y un pancreatograma, en el que se observó una dilatación sacular de $3 \mathrm{~cm}$ asociado con un cálculo de 15 $\mathrm{mm}$ en la cabeza del páncreas; se pasó una guía hidrofílica alojándola en el ducto pancreático y, posteriormente, se realizó una esfinterotomía pancreática. Se retiró el papilótomo, sobre la guía se montó el catéter del Spyglass, se logró acceder al conducto pancreático en el que se observó una gran dilatación y la presencia de un cálculo ovoide de aspecto blanquecino (Figura 2). Se pasó una sonda láser Holmium (Figura 3) y se fracturó el cálculo en múltiples fragmentos, se extrajo

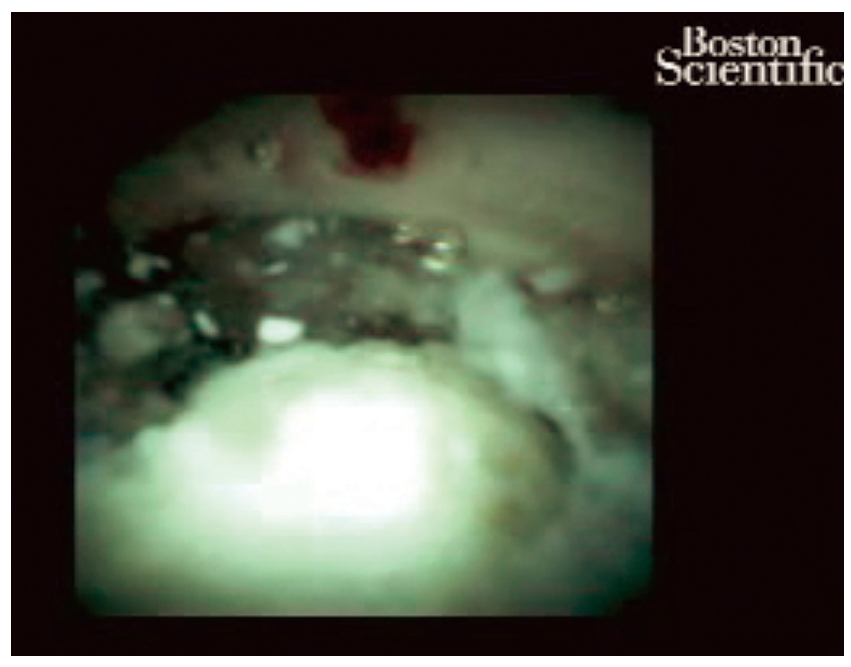

Figura 2. Cálculo pancreático de aspecto blanquecino característico del páncreas.

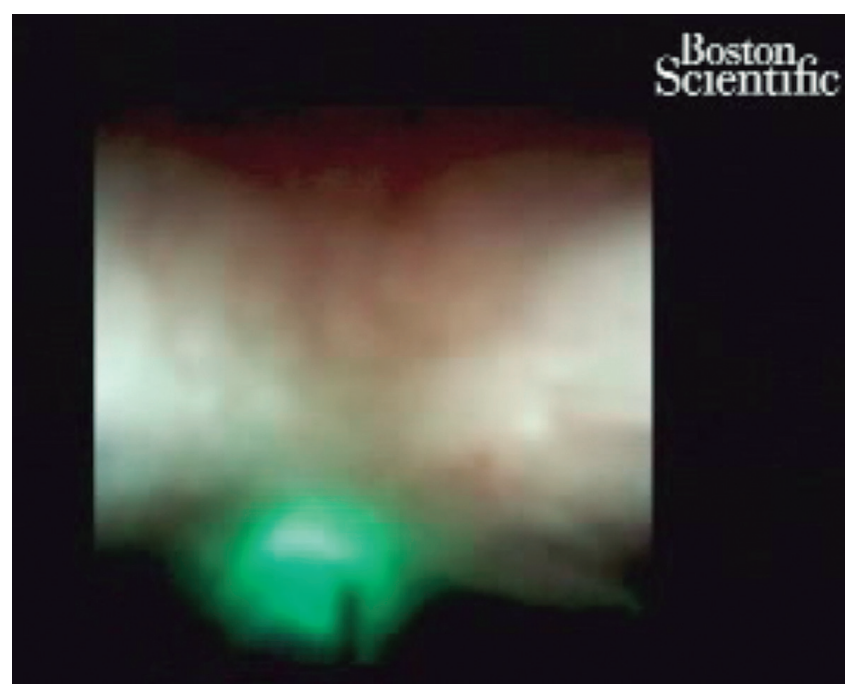

Figura 3. El láser (luz verde) señala en dirección al cálculo que se va a fracturar.

su totalidad con la canastilla y se dejó un stent de $7 \mathrm{f}$ para garantizar el drenaje de residuos adicionales. Se mantuvo durante observación clínica por 24 horas, en las que tuvo una adecuada evolución. Durante los controles ambulatorios el paciente manifestó un adecuado control de síntomas, sin requerimiento de uso de analgésicos hasta la fecha debido a la resolución del dolor.

\section{DISCUSIÓN}

La pancreatoscopia es un procedimiento novedoso el cual permite realizar una endoscopia dentro del conducto pancreático con fines diagnósticos y terapéuticos (9). Es eficaz 
especialmente en patologías pancreáticas de difícil diagnóstico y tratamiento en fases tempranas como la pancreatitis crónica, pancreatitis recurrente y manejo de colecciones líquidas (10). En estos momentos son pocos los centros en Colombia que pueden ofrecerla a sus pacientes, por lo cual consideramos fundamental dar a conocer la posibilidad de implementar este tipo de intervenciones. Este es el primer caso reportado en Colombia del manejo exitoso de un paciente con pancreatitis crónica y cálculo pancreático con colangiopancreatoscopia directa tipo Spyglass.

Consideramos que el enfoque terapéutico más apropiado para nuestro país, teniendo en cuenta las limitaciones económicas inherentes sobre los servicios de salud, sería realizar primero una CPRE e intentar realizar la extracción del cálculo pancreático con los accesorios convencionales (canastilla o balón). Luego de esto y con el fin de evitar el desarrollo de complicaciones posteriores, principalmente pancreatitis, siempre se debe dejar alojado un stent pancreático (11). Solo en los casos en que este procedimiento no tenga éxito, se debe considerar realizar pancreatoscopia y litotricia láser a través del sistema Spyglass.

\section{CONCLUSIÓN}

Se presenta el primer caso reportado en Colombia del uso de Spyglass para tratar a un paciente con un cálculo pancreático grande asociado con un pseudoquiste. Coincidimos en que este método, aunque es muy costoso, es útil para el tratamiento de este tipo de pacientes, que son de muy difícil manejo.

\section{REFERENCIAS}

1. Alexandrino G, Lourenço L, Rodrigues CG, Horta D, Reis J, Canena J. Pancreatoscopy-guided laser lithotripsy in a patient with difficult ductal stone. Endoscopy. 2018;50(6):E130-E131. doi: 10.1055/a-0574-2278.

2. Bekkali NL, Murray S, Johnson GJ, Bandula S, Amin Z, Chapman $\mathrm{MH}$, et al. Pancreatoscopy-Directed Electrohydraulic Lithotripsy for Pancreatic Ductal
Stones in Painful Chronic Pancreatitis Using SpyGlass. Pancreas. 2017;46(4):528-30. doi: 10.1097/ MPA.0000000000000790.

3. Tsuchiya T, Itoi T. Diagnostic and therapeutic cholangiopancreatoscopy for pancreatobiliary diseases. Nihon Shokakibyo Gakkai Zasshi. 2017;114(8):1423-35. doi: 10.11405/nisshoshi.114.1423.

4. Pereira P, Peixoto A, Andrade P, Macedo G. Peroral cholangiopancreatoscopy with the SpyGlass ${ }^{\circledast}$ system: what do we know 10 years later. J Gastrointestin Liver Dis. 2017;26(2):165-70. doi: 10.15403/jgld.2014.1121.262.cho.

5. Woo YS, Lee JK, Oh SH, Kim MJ, Jung JG, Lee KH, et al. Role of SpyGlass peroral cholangioscopy in the evaluation of indeterminate biliary lesions. Dig Dis Sci. 2014;59(10):2565-70. doi: 10.1007/s10620-014-3171-x.

6. Parsi MA. Peroral cholangioscopy in the new millennium. World J Gastroenterol. 2011;17(1):1-6. doi: 10.3748/wjg. v17.i1.1.

7. Moon JH, Terheggen G, Choi HJ, Neuhaus H. Peroral cholangioscopy: diagnostic and therapeutic applications. Gastroenterology. 2013;144(2):276-82. doi: 10.1053/j. gastro.2012.10.045.

8. Chen YK, Pleskow DK. SpyGlass single-operator peroral cholangiopancreatoscopy system for the diagnosis and therapy of bile-duct disorders: a clinical feasibility study (with video). Gastrointest Endosc. 2007;65(6):832-41. doi: 10.1016/j.gie.2007.01.025.

9. Chen YK. Preclinical characterization of the Spyglass peroral cholangiopancreatoscopy system for direct access, visualization, and biopsy. Gastrointest Endosc. 2007;65(2):30311. doi: 10.1016/j.gie.2006.07.048.

10. Ang TL. Chronic pancreatitis with pancreatic duct stricture and calculi treated by fully covered self-expandable metal stent placement and intraductal pancreatoscopy-guided laser lithotripsy. Endoscopy. 2017;49(6):E145-E146. doi: 10.1055/s-0043-105570.

11. Parbhu SK, Siddiqui AA, Murphy M, Noor A, Taylor LJ, Mills A, et al. Efficacy, Safety, and Outcomes of Endoscopic Retrograde Cholangiopancreatography With Per-Oral Pancreatoscopy: A Multicenter Experience. J Clin Gastroenterol. 2017;51(10):e101-e105. doi: 10.1097/ MCG.0000000000000796. 